\title{
Improving Reliability and Reducing Power Loss in Power Distribution Network by Determining Optimal Location and Size of Capacitor Banks
}

\author{
M. Nadjafi ${ }^{1 *}$ and M. Hajivand ${ }^{1,2}$ \\ 1. Aerospace Research Institute (Ministry of Science, Research and Technology); Department of Aerospace \\ Management, Law and Standards; Tehran, P.O.B 14665-834, Iran
}

2. Young Researchers and Elite Club, Borujerd Branch, Islamic Azad University, Borujerd, Iran

\begin{abstract}
The use of capacitor banks in distribution system has many outstanding usages include improving the power factor of a system, voltage profile, and reliability besides the reducing of the power flow losses of the component's reactive due to the compensation. These benefits depend greatly on how capacitors are placed in the distribution system. Hence, in order to achieve the high reliable construction, switching capacitor has been placed to improve the main challenges of the network designing (reliability and reduce power loss) in the radial distribution system. As regards, the importance of the reliability and power losses are ignored in the distribution networks; the aim of this paper is primarily to establish an objective function for the parallel optimization of these aforementioned parameters. In the simulation process, ten parameters have been compared, which are: System Average Interruption Frequent Index (SAIFI) and its cost, System Average Interruption Duration Index (SAIDI) and its cost, power loss and its cost, the installed capacity and it's cost and values of two objective functions. Honey-bee mating optimization (HBMO) algorithm has been used to solve this problem. Then, the developed technique has been used on the IEEE standard distribution network as a problem-solving system.
\end{abstract}

Keywords: Capacitor placement; Honey-Bees Mating Optimization; Optimal Location; Power loss; Reliability

$\begin{array}{ll}\text { Nomenclature and Units } \\ \text { BFOA } & \text { Bacterial Foraging Optimization Algorithm } \\ C O A & \text { Cuckoo Optimization Algorithm } \\ D E & \text { Differential Evolution } \\ D N & \text { Distribution Network } \\ D R D L F & \text { Dimension Reducing Distribution Load Flow } \\ G A & \text { Genetic Algorithm } \\ H B M O & \text { Honey-Bees Mating Optimization } \\ L_{i(\alpha)} & \text { Average Load } \\ L S I & \text { Loss Sensitivity Index } \\ N_{i} & \text { Number of Networks } \\ O F & \text { Objective Function } \\ P_{d i} & \text { Active Power Generation } \\ P_{g i} & \text { Active Power Loads } \\ P_{i j} & \text { Active Power from } i \text { to } j \text { bus } \\ Q_{C} & \text { Active Bus Loads } \\ Q_{d i} & \text { Reactive Power Generation } \\ Q_{g i} & \text { Reactive Power Loads } \\ Q_{L} & \text { Reactive Bus Loads } \\ R C G A & \text { Real Coded Genetic Algorithm } \\ r_{S} & \text { Average Time Duration Outage } \\ S A I D I & \text { System Average Interruption Duration Index } \\ S A I F I & \text { System Average Interruption Frequency Index } \\ U C & \text { Unit Commitment } \\ U_{S} & \text { Annual Average Duration Outage } \\ & \\ & \end{array}$

${ }^{*}$ Corresponding author: M. Nadjafi, PhD, Assistant Professor in Aerospace Engineering; E-mail address:m.nadjafi@ari.ac.ir

$\begin{array}{ll}V_{i} & \text { Measured Voltage } \\ V S I & \text { Voltage Stability Index } \\ Y_{i j} & \text { Bus Matrix Admittance } \\ \delta_{i} & \text { Phase Angle } \\ \theta_{i j} & \text { Admittance Angle } \\ \lambda_{i} & \text { Failure Rate }\end{array}$

\section{Introduction}

In a power system, generators generate reactive power and transfer through transmission lines to consumers. The reactive power occupies a large amount of generator capacity. Therefore, using parallel capacitors can be used to generate reactive power near consumers, to release the existing capacity of the line and equipment to improve the voltage and reduce power losses. It is well-known that parallel capacitors are the most economical source of reactive power generation ${ }^{[1]}$. Most useful tools and techniques have been developed and are used in order to optimize the reliability associated parameters related to the power distribution network issues. Based on the type of the optimization and indexes used in these type of problem analysis, generally, the methods based on the metaheuristic approaches are widely used. In this regard, in order to allocate optimal location on capacitor banks 
and its switches in DN, algorithms based on evolutionary techniques have been developed ${ }^{[2][3]}$. As well as, the techniques based on the novel Genetic Algorithm have been developed to optimal sizing and locating the shunt capacitors on DN systems ${ }^{[4][5]}$. In this context, a genetic algorithm based on a fuzzy multi-objective method is proposed to determine the optimal values of the shunt and switching capacitors in order to improve the voltage profile and minimize power losses. The superiority of the pre-existing fuzzy technique has been proven by experiment on a 69-bus standard test system and the definition of three levels of load on this network ${ }^{[6]}$.

In order to improve the quality of power in a network, some research and study have been done to find the location and determination of the optimal capacity of the shunt capacitor. To this end, the new target function is used to solve the target function (which is a function of the cost of power losses, energy losses, and capacitor bank charges), and the constraints (voltage range, number and capacity of installed capacitors). The simulations results are compared with another version of the genetic algorithm ${ }^{[7]}$. On the other hand, function-based functions on capacitor cost to minimize power and energy losses and improve the voltage profile have proposed ${ }^{[8]}$. Also, a novel algorithm based on a genetic algorithm is used to solve this problem by considering the voltage stability limit. The 34-bus standard test system is used as a sample network and simulation results are provided by different scenarios. Furthermore, in the radial distribution systems, a new approach based on the scheduling of long term in order to find out the optimum allocating of the capacitor bank with the objective function of minimizing the losses of power has been developed using the Bacterial Foraging Optimization Algorithm (BFOA). The Loss Sensitivity Index (LSI) and Voltage Stability Index (VSI) are included in the target function. In the proposed model, load variations from low frequency $(50 \%)$ to high $(160 \%)$ on a distributed network of 34 and 85 IEEE bus is performed ${ }^{[9]}$ and the same is done by the with the Cuckoo Optimization Algorithm (COA) ${ }^{[10]}$.

On the other hand, the methods based on analytical solutions are the other useful techniques to treat related issues. In this regard, the techniques to find and detect the direction of the capacitors in $\mathrm{DN}$, besides the point of spatial perspective have been developed in ${ }^{[11][12]}$ to control the switching process of the capacitors in the power networks. These transmissions are transmitted through the network along with the transmission elements, and so on in other places away from capacitors, such as load terminals ${ }^{[13]}$. In this field, a computationally efficient methodology is proposed for the optimal location and sizing of static and switched shunt capacitors in radial distribution systems ${ }^{[14]}$. Similar work has been performed in order to the improvement of the voltage stability index by optimal capacitor location using an analytical technique that is strongly sensitive to voltage collapse in distribution networks ${ }^{[15]}$. The proposed technique is accomplished and has been tested on the 34 and 69 IEEE standard test system in ${ }^{[16]}$. Accordingly, two optimization models are proposed for obtaining optimal capacitor locations for maintaining the voltage profile in distribution networks. First, the problem of locating the element as a power transmission problem is correlated with an innovative mathematical representation of the formation of the voltage profile. Then, the problem of the location of the capacitor is modeled and solved. Ramos and his colleagues focused on the optimal location of capacitor banks based on the average curve of daily load variations in the unbalanced distribution network ${ }^{[17]}$. The simulation results are implemented on IEEE standard networks and compared to the results in the background ${ }^{[18]}$, two main goals are the calculation of the reactive power demand and minimization of the cost.

Another suggested class of analysis methods is the Metaheuristic techniques ${ }^{[19]}$. In the heuristic approach, a practical solution technique is proposed for the capacitor placement problem that is easy to implement ${ }^{[20]}$, also, other most heuristic algorithms in order to find the optimal placement of capacitors and the rating of them in DN systems are studied in detail in ${ }^{[21][22][23]}$.

Based on studies done, the use of capacitor banks in distribution system has many outstanding usages include improving the power factor of a system, voltage profile, and reliability besides the reducing of the power flow losses of the component's reactive due to the compensation. These benefits depending on how the capacitors are placed in the distribution system. Hence, in order to achieve highly reliable construction, switching capacitor has been placed to improve reliability and reduce power loss in the radial distribution system. The optimality of the two parameters of power loss and reliability is one of the main challenges for network designers. As regards, the importance of the reliability and power losses are ignored in the distribution networks; this paper primarily focuses on the parallel optimization of these parameters. The two parameters of the System Average Interruption Frequency Index (SAIFI) and System Average Interruption Duration Index (SAIDI) as a measure of reliability improvement have been investigated. Since the HBMO is part of the latest particle intelligence techniques, this paper has been used as a problem-solving technique and a study on the IEEE standard distribution network with scenario-based designs based on the number of capacitor banks has been accommodated.

The remainder of this paper is structured as follows: Section 2 presents the theoretical basics about reliability and losses in distribution networks. Formulating Structure for the objective function and constraint relations are proposed in section 3 . 
The concept of the proposed HBMO algorithm has been presented in section 4 . Based on the proposed algorithm, the simulation results are provided in Section 5 and the last part is about the conclusion and future work.

\section{Theoretical Basics}

A distribution network (DN) as the last section of a power system in each country has the widest variety of equipment and the most extensiveness, both of which may lead to many problems in the system. Using a wide variety of equipment in a great number increases the probability of failures and power outage which decreases the system reliability. Since DNs are directly connected to the consumers with no mediates, the current value is high and the voltage value is low and in case the network is excessively extended the high current will rise even more. Therefore, resistive losses (or $\mathrm{RI}^{2}$ losses) is very high in DNs, especially in Iran's power grid. The mentioned problem will be doubled when the $\mathrm{DN}$ is designed in the form of a radial topology. The DN designers do not have various choices to solve and/or reduce the resistive losses because economic and environmental issues should also be taken into account while improving and solving the technical problems. Thereby, most of the network designers suggest using capacitor banks to overcome the dominant problems of DNs [24].

\subsection{Distribution Network Reliability}

Regarding that the proposed objective function in this paper consists of reliability parameters, at first, the importance of reliability in power systems, especially in DNs, is addressed. The following are the importance of risk studies in a $\mathrm{DN}^{[25]}$ :

The dominance of calculating risk index in a $\mathrm{DN}$ is not less than that of generation and transmission networks because the distribution section has a considerable share of the power outage. Also, the reconstruction of power systems is added to this issue (calculation of losses). In a reconstructed environment, economic factors play a noticeable role and are not less important than technical factors.

The aims of studying reliability in DNs include: Determination of network's reliability and the level of customers satisfaction that contains the frequency of shortterm and long-term outages, outage duration, and the number of interrupted customers; Reliability improvement; A basis for development and design of the network; Determining the characteristics of the implementation of operation-based regulations; and Maintenance scheduling and Unit Commitment (UC) or resource allocation.

On the other hand, the reliability of a DN is expressed in terms of reliability indices. The reliability evaluation results of DNs are presented in the form of load nodes or overall system indices. Reliability indices of load nodes include the average rate of failure occurrence $\lambda$ (failure/year), the average time duration of outage $r$ (hour), and the annual average duration of outage U (hour/year) ${ }^{[26]}$ :

$\lambda_{s}=\sum_{i \in A} \lambda_{i}($ failure $/$ year $)$

$U_{s}=\sum_{i \in A} \lambda_{i} \cdot r_{i}($ hour $/$ year $)$

$r_{s}=\frac{U_{s}}{\lambda_{s}}($ failure $/$ year $)$

Where $\lambda_{i}$ is the rate of failure occurrence at the $i$-th mode, and $r_{i}$ is the required time for supply restoration to the considered load nodes after a failure occurs at the $i$-th mode.

In order to complete the required equations in the reliability analysis of the distribution networks, the following three indices are utilized in this paper to evaluate the reliability improvement trend. First, the System Average Interruption Frequency Index (SAIFI); this index is defined to obtain information on the average number of system interruptions for each customer in a specific area:

$S A I F I=\frac{\sum_{i=1}^{n} U_{i}}{\sum_{i=1}^{n} N_{i}}$

Second, the System Average Interruption Duration Index (SAIDI); this index commonly addresses the number of minutes the customers are interrupted, and it is used to avoid the information employed regarding the average interruption duration of each customer.

$S A I D I=\frac{\sum_{i=1}^{n} N_{i} U_{i}}{\sum_{i=1}^{n} N_{i}}$

\subsection{Distribution Network Losses}

One of the important parameters in a power system is the system losses, which determines the optimality, efficiency, and long-term operating costs of the system. Energy loss is obtained, in fact, from the summation of the instantaneous values of power losses and is the difference between the consumed power and the generated power by the customer. That part of the electrical energy is not converted to useful work is called losses, therefore the losses formed due to the low efficiency of power equipment is also included in this part of the energy. Two topics in the field of losses that are worth mentioning are ${ }^{[24-25]}$ : First, the losses from the electrical energy distribution companies' point of view; this power loss is the difference between the delivered energy and the output energy. If the power loss is studied from an economic standpoint, then it is the difference between the purchased and sold energies. As a result, only the occurred losses in the power systems are important from the standpoint of these companies. However, if the power loss is also taken into account as a factor in these companies' policy, then the 
economic price of the losses should be considered in the calculations as well. Second, the imposed costs by power losses; the high value of the annual costs a distributor suggests that the loss reduction leads to considerable benefits. This value should be annually calculated for every distribution company. Although the removal of all the losses is impossible, the removal of a part of the annual losses cost may result in a dramatic cost saves to justify the investments for improving the efficiency of networks.

\section{Formulating the Proposed Method}

In this section, in order to improve the reliability of the system and reduce power loss in the radial distribution system, required equations and relationships based on Object Function (OF)and constraints are separately derived and proposed which are discussed bellows.

\subsection{Objective Function}

One of the main challenges of the designers of distribution systems is trying to improve reliability and reduce power losses. To accomplish this goal in this article, the cost objective function on the basis of the minimizing power loss cost (CLOSS) and maximizing reliability has been formulated. To do this, the Cost of System Average Interruption Frequent Index (CSAIFI) and Cost of System Average Interruption Duration Index (CSAIDI) have been used as the main index of the reliability indices. The unit installation has applied the cost of installation and operation of Capacitor Banks (CWF) to the system. Then the proposed cost objective function is as following,

COF $=$ CCAP + CLoos + CSAIDI + CSAIFI

\subsection{Constraint Functions}

Conditions of the problem are mainly the limitations of utilization and the problems that must be considered in relation to power quality. Our problem involves a series of equal and unequal constraints. The unequal constraints are related to the bus voltage, power-flow and the maximum injection power to each bus which should be within a certain range. Equivalence also involves load flow equations, which must be true for all variables in the system. In the following, each of these constraints will be investigated along with the corresponding equations.

Load Flow Constraint: Load flow is the first step in optimal capacitor placement. However, the observance of the load flow in solving power system problems is obvious, but its expression is indicative of its importance. The load flow relations for active power and reactive power are formulated as follows ${ }^{[27]}$ :
$P_{g i}-P_{d i}-V_{i} \sum_{j=1}^{N} V_{j} Y_{i j} \cos \left(\delta_{i}-\delta_{j}-\theta_{i j}\right)=0$

$Q_{g i}-Q_{d i}-V_{i} \sum_{j=1}^{N} V_{j} Y_{i j} \sin \left(\delta_{i}-\delta_{j}-\theta_{i j}\right)=0$

$P_{g i}, Q_{g i}:$ Active and Reactive power generation in $i$ bus.

$P_{d i}, Q_{d i}$ : Active and Reactive loads in $i$ bus.

$V_{i}, \delta_{i}$ : Measuresand the Voltage phase angle of $i$ bus.

$Y_{i j}, \theta_{i j}$ : Bus Matrix admittance and admittance angle.

Voltage and Power Constraints: With the location, the value of the voltage of each bus should be changed within a reasonable range, the bus voltage below the lowest defined voltage means the ineffectiveness of the proper distribution of capacitor banks, in terms of size and capacity, and between the bus and the value Most of the voltage above the defined threshold creates overvoltage problems. Voltage change in a reasonable range, as one of the objectives of the capacitor location, is specified as follows ${ }^{[28]}$ :

$V_{i}^{\min } \leq V_{i} \leq V_{i}^{\max } ; i=1,2, \ldots, N$

Where $V_{i}$ is the $i$-th bus voltage.

The range of losses between the bus and the distribution network is calculated from the Equation (11):

$P_{i j}^{\min } \leq P_{i j} \leq P_{i j}^{\max } ; i=1,2, \ldots, N$

$P_{i j}$ is the active power from bus $i$ to $j$.

Installed Capacity Constraint: The maximum capacity of the installed capacitor should be less than or equal to the reactive load in the network, which means that the installed capacitor must not be so large that the network goes from the self-to-capacitive mode and shows capacitive behavior. This concept is given by ${ }^{[29]}$ :

$Q_{C}^{\text {Total }}=\sum_{i=1}^{N_{C}} Q_{i} ; Q_{C}^{\text {Total }} \leq Q_{L}$

$Q_{C}^{\text {Total }}$, the VAR connected loop by capacitor banks for radial distribution network; and $Q_{L}$ is the Reactive loads in the redial distribution network.

\section{Solving the Problem by Proposed HBMO Algorithm}

One of the oldest and earliest methods that have been developed in the analysis of the optimization problems is the HBMO technique. This technique has been developed based on the sociality treatment of the insect in his colony, community, and pro-social behaviors based. The community of the colony of insects contains three episodes: The Queen (to fertility and reproduction of the new groups), Explorers (to discover and explore the best ways of possibility), and the Workers (slaves for porterage). HBMO as many other 
heuristic algorithms is made on the basis of the real life and relation of social creatures in particular on bees. Any of the Honey-Bee Mating Optimization algorithms contain the following summarized process ${ }^{[30]}$ :

1. Initially, the queen of the colony searching for his kind to find the best and most suitable case for sperm theca as likely as possible from the male bees.

2. Secondly, the new colonies generated from the mating of the queen and stronger male bees.

3. Workers try to search the broods in order to find the new generation for presenting the new queen. (this step is a trial and error process).

4. The fitting function of the workers is arranged according to the progress made in the bees' generation.

5. The superior bee (competition between young and old queens) to replace in the queen position and perform the next mating process.

The starting process is the queen of the colony searching for his kind to find the best and most suitable case for sperm theca as likely as possible from the male bees is performed by the solving of the following probability equation:

$\operatorname{pr}(Q, D)=\exp \left(\frac{-\Delta(f)}{s(t)}\right)$

In the above equation, $\operatorname{Pr}(Q, D)$ is the drone's sperm adding probability $(D)$ in to the queen's sperm theca $(Q)$, also, $S(t)$ is the queen speed at time t, and $\Delta(f)$ is the drone's $(f(D))$ and the queen's $(f(Q))$ fitness in the term of absolute differential. It is evident that over time, the queen's energy and velocity decreases and the deterioration rates of these two parameters are expressed using the following equations:

$S(t+1)=\beta \times \mathrm{S}(\mathrm{t})$

$E n(t+1)=E n(t)-\gamma$

Here, $\beta$ is a constant and its value is varied between 0 and 1 , and $\gamma$ is the deterioration rate of the queen's energy and velocity per trip. Queen's speed is selected probabilistically at the start of the process. It should be noted that the amount of the Queen's speed is one of the initial inputs. Further discussion of this algorithm and its details are given in some references. ${ }^{[30-31-32]}$

So far, the concept of optimal capacitor placement problem, distribution network functions and relations, and the HBMO algorithm has been presented. In the following, the capacitor placement problem solution by HBMO algorithms is presented based on the provided flowchart in Fig.1.

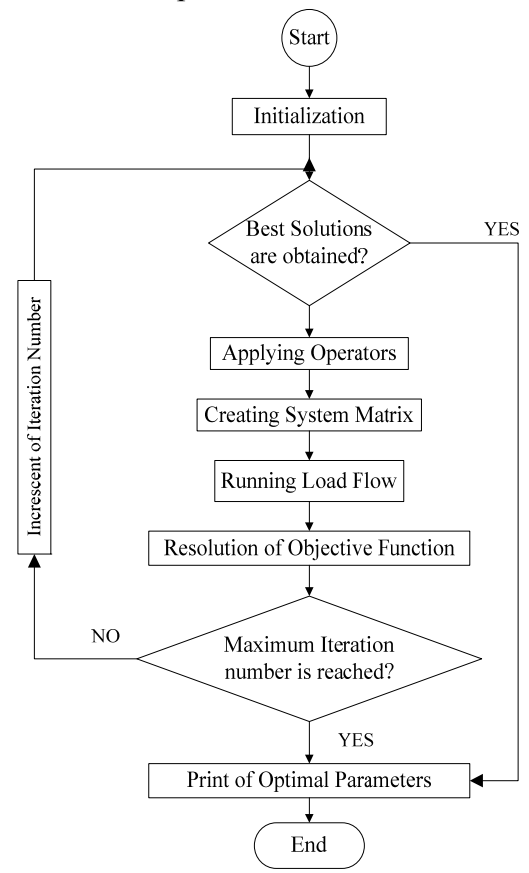

Fig.1. Flowchart of the improving reliability indices based on the proposed HBMO approach

\section{Simulation Results}

Simulation has been performed on the 36-bus radial distribution network. Fig.2 shows a single line diagram of the test system.

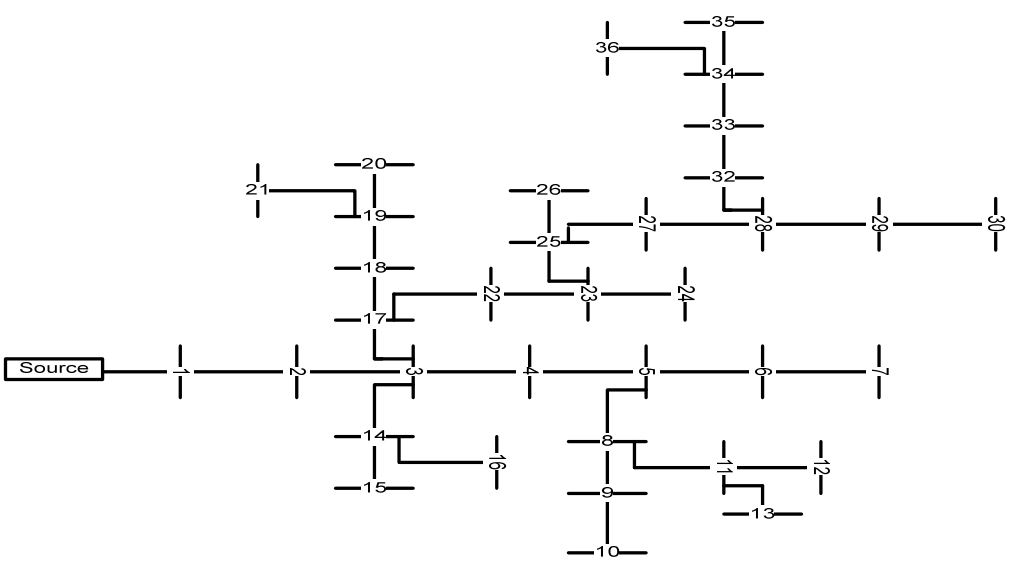

Fig.2. Diagram of the 36-bus single line system 
International Journal of Reliability, Risk and Safety:

Theory and Application / Vol. 1, No. 1, 2018

www.IJRRS.com

In simulation process, ten parameters have been compared: SAIDI (in hours per the fault in year), CSAIDI (in million Tomeans), SAIFI (fault per year), CSAIFI (in million Tomeans), power loss (in kW), class (in million Tomeans), the installed capacity and its cost, parametric function and cost

function (in million Tomeans). Placement process starts from one unit and continues until the network is reaching saturation.

Table 3 illustrates the results of the placement in the test system.

Table 3. Objective Functions Parameters Values

\begin{tabular}{|c|c|c|c|c|c|c|}
\hline $\begin{array}{l}\text { The number of } \\
\text { CAP }\end{array}$ & Case & $\begin{array}{l}\text { Objective } \\
\text { function }\end{array}$ & $\begin{array}{l}\text { Installed } \\
\text { Capacity }\end{array}$ & Power loss & SAIFI & SAIDI \\
\hline \multirow{2}{*}{1} & Parameter & 2.9040 & 150 & 94.7475 & 21.8281 & 15.4707 \\
\hline & cost & 52.5250 & 49.2955 & 24.8993 & 17.4622 & 5.2333 \\
\hline \multirow{2}{*}{2} & Parameter & 2.8516 & 175 & 91.7484 & 21.5792 & 15.2890 \\
\hline & cost & 56.7388 & 10.1920 & 24.1115 & 17.2634 & 5.1718 \\
\hline \multirow{2}{*}{3} & Parameter & 2.7728 & 295 & 87.4540 & 21.1847 & 14.9952 \\
\hline & cost & 68.4007 & 23.3976 & 22.9829 & 16.9477 & 5.0724 \\
\hline \multirow{2}{*}{4} & Parameter & 2.6844 & 520 & 82.2154 & 20.7775 & 14.7060 \\
\hline & cost & 68.4390 & 25.2362 & 21.6062 & 16.6220 & 4.9745 \\
\hline \multirow{2}{*}{5} & Parameter & 2.7264 & 750 & 82.5871 & 21.1873 & 15.0168 \\
\hline & cost & 71.5185 & 27.7850 & 21.7039 & 16.9499 & 5.0796 \\
\hline
\end{tabular}

By considering the results of table 3, it can be argued that it has the necessary technical and economic feasibility of installing four but installing more than this number, the destruction of the objective function parameters. In fact, despite the increase in installed capacity of capacitors response of five evidence of the worse outcome. Optimal location and size of the placed units have been listed in Table 4 .

Table 4. Optimal Location and Size Of The Placed Units

\begin{tabular}{c|c}
\hline Scenario & Location (size) \\
\hline 1 & $19(150)$ \\
\hline 2 & $33(50) ، 19(125)$ \\
\hline 3 & $33(20) ، 2(125) ، 15(150)$ \\
\hline 4 & $2(160) ، 18(80) ، 21(80) ، 33(200)$ \\
\hline
\end{tabular}

Based on the results of Table 4, it can be claimed that bus 33 is the most likely location for the installation of Capacitor Banks. In this section, results of each parameter in five scenarios are compared. Fig. 3 shows the results of SAIDI in five scenarios.

By considering results of Fig.3, it is clear that SAIDI values declined during the first four scenarios which this process is demolished by increasing the number of units to five units. Fig.4 illustrates the cost of SAIDI.

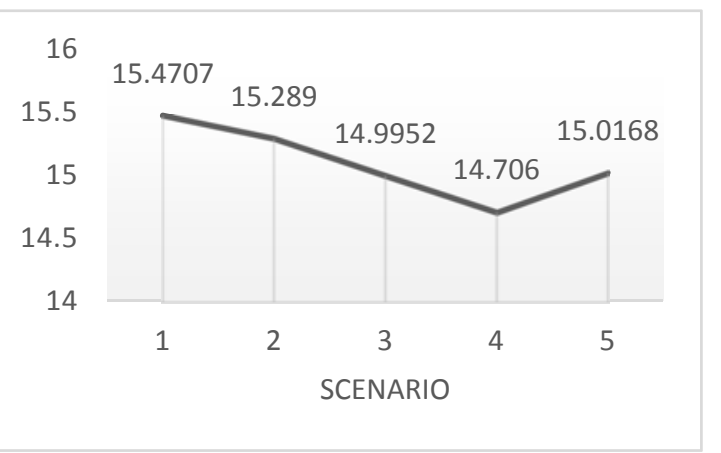

Fig.3. SAIDI comparison

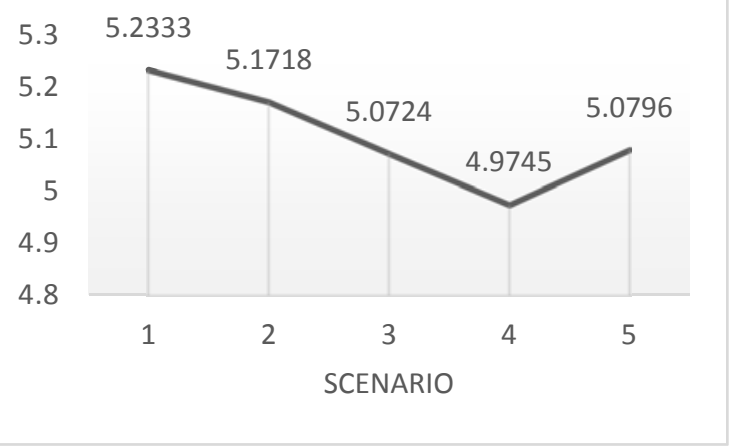

Fig.4. CSAIDI comparison

Based on Fig.4, it is clear that the behavior of cost of SAIDI is similar to SAIDI. SAIFI of five scenarios is demonstrated in Fig. 5. 


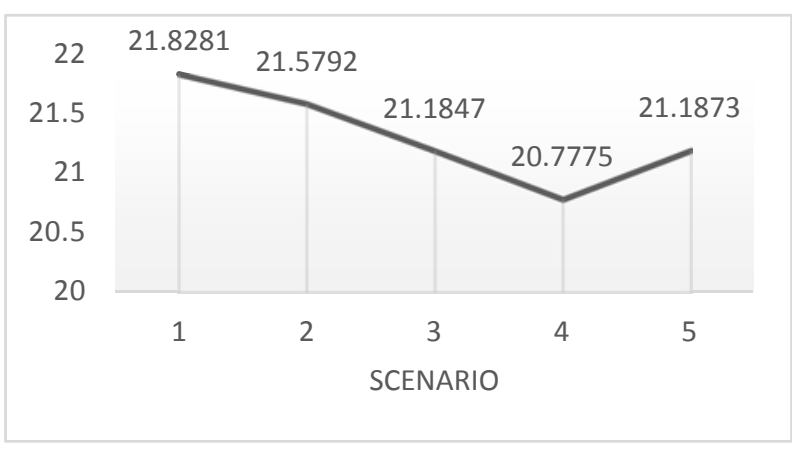

Fig. 5. SAIFI comparison

From Fig. 5, although the behavior of SAIFI is similar to SAIDI but with a less steep slope of decline is less than the value being reduced. Cost of SAIFI is visible in Fig.6.

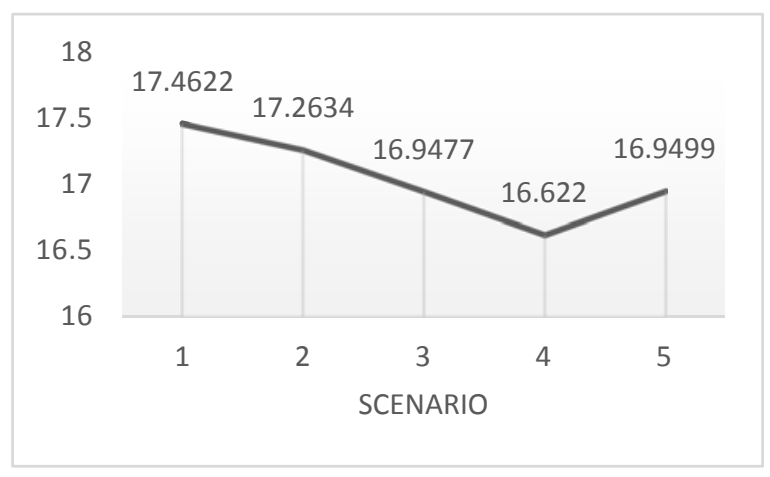

Fig.6. CSAIFI comparison

From Fig.6, CSAIFI behavior is similar to SAIFI. Fig.7 shows the power loss of the five scenarios.

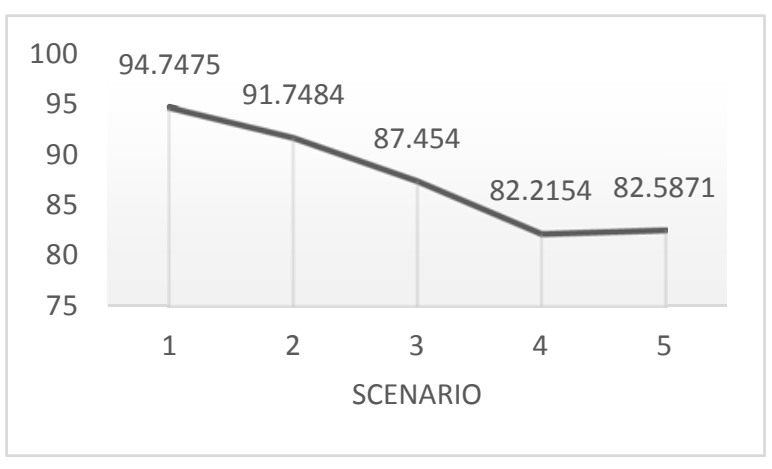

Fig.7. Power losses comparison

In this figure, the amount of power loss after the fourth scenario remains almost constant. Cost of power loss is visible in Fig.8. Furthermore, Fig.9 shows a comparison of capacitor banks sizes in five scenarios.

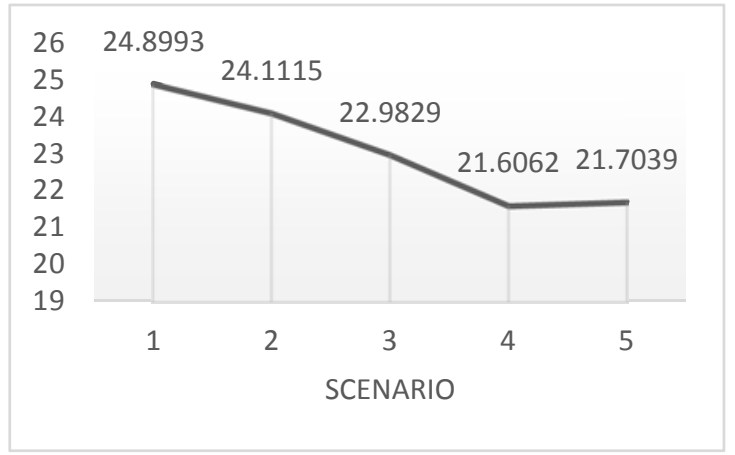

Fig.8. Power loss cost comparison

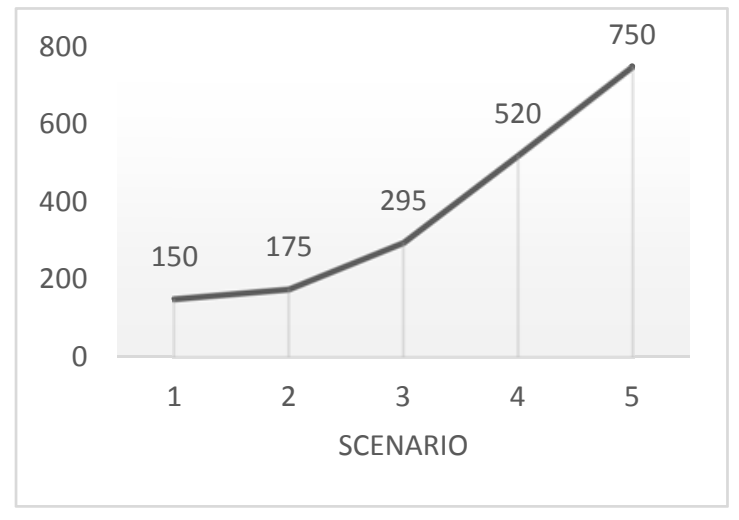

Fig.9. Capacity of the installed unit comparison

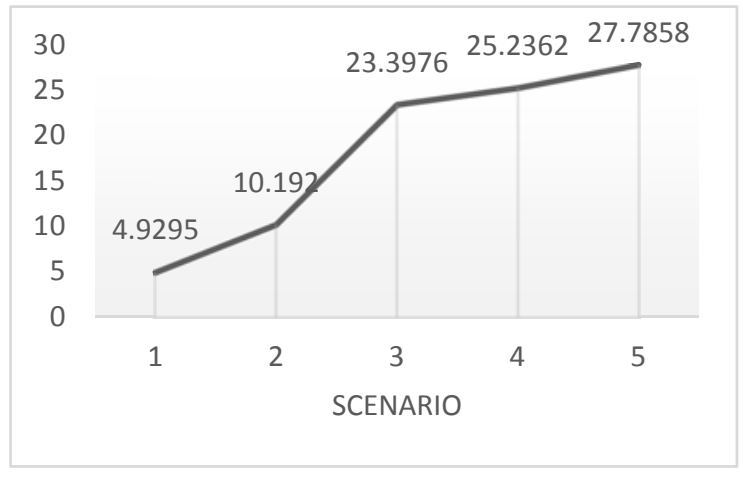

Fig.10. Cost of the installed unit comparison

Similar behavior of the cost and capacity of installed capacity is quite obvious. Fig.11 and Fig.12 provide parametrically and cost objective functions, respectively. 
www.IJRRS.com

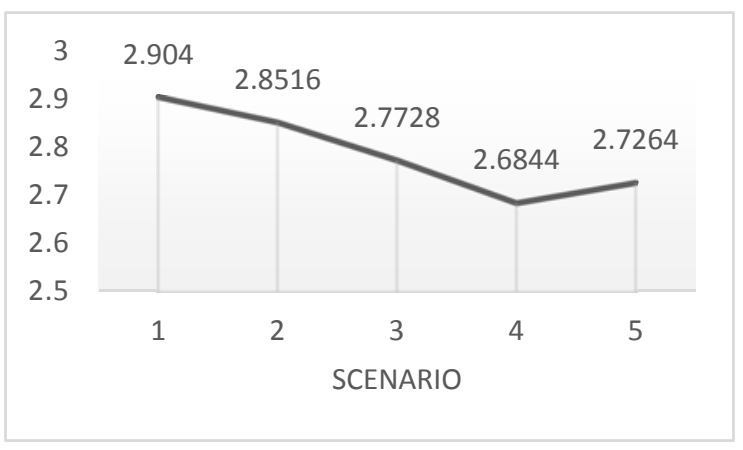

Fig.11. Parametric function comparison

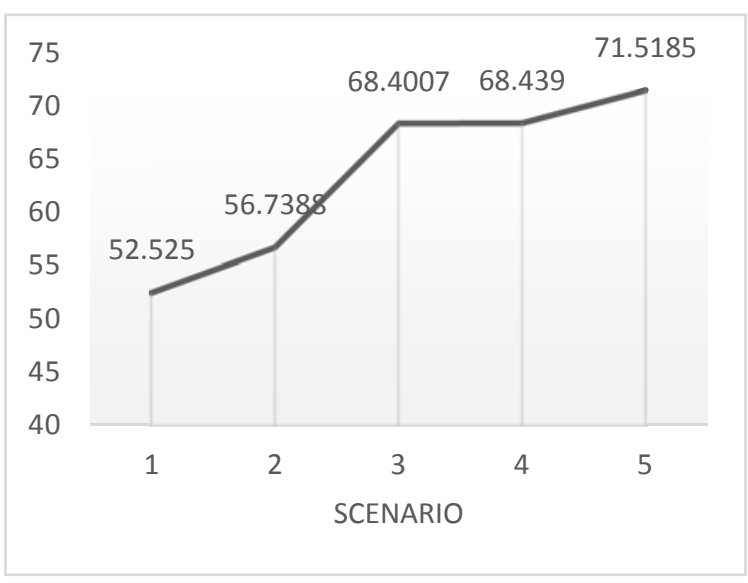

Fig.12. Cost function comparison

\section{Conclusions and Future Work}

Shunt capacitor banks installation in distribution systems leads to; reduction of power (energy) loss, raising the availability of feeders, improvement profile of voltage and correction of the power factor. The main problem in installing capacitor banks is finding optimal location and size so that the annual cost of system minimized. In this paper, in order to optimization aforementioned problem, two main challenges related to the distribution network installing capacitor banks are: the improvement of reliability (due to the variety and number of equipment, the structure of the entity and the close relationship with consumers) and the minimization of power losses (due to the high level of current flow in the distribution network) are considered. To achieve this goal, we introduce an objective function for the HBMO algorithm implemented on the 36-bus IEEE standard that could cover either the reliability indices or the power loss parameter. These results indicate that increasing the number of capacitors not only increase reliability and reduce power losses, but also increase the cost and complexity of the network distribution structure. In the future work, the authors try to use of enhanced HBMO algorithm, to improve the capabilities of the HBMO algorithms. Hence, Coordinate Distributed
Generation (DG) placement with capacitors is overlapping in terms of minimizing power losses and improving network reliability will be considered since it is one of the topics of interest to network designers.

\section{References}

[1]. Chiou J.P. and Chang C.F., Development of a novel algorithm for optimal capacitor placement in distribution systems. International Journal of Electrical Power \& Energy Systems, 73: pp. 684-690, 2015.

[2]. Ataei R.,A.H.M. and Hooshmand R., Optimal capacitor placement in actual configuration and operational conditions of distribution systems using RCGA. Journal of Electrical Engineering, 58(4): pp. 189-199, 2007.

[3]. HaghifamM.R,. and Malik O., Genetic algorithm-based approach for fixed and switchable capacitors placement in distribution systems with uncertainty and time varying loads. IET generation, transmission \& distribution, 1(2): pp. 244-252, 2007.

[4]. Swarnkar A., Gupta N., and Niazi K.,Optimal placement of fixed and switched shunt capacitors for large-scale distribution systems using genetic algorithms. in Innovative Smart Grid Technologies Conference Europe (ISGT Europe), IEEE PES. 2010.

[5]. Neelima S., and Subramanyam P., Efficient optimal sizing and allocation of capacitors in radial distribution systems using drdlf and differential evolution. International Journal on Electrical and Power Engineering, 2(03), 2011.

[6]. Das D., Optimal placement of capacitors in radial distribution system using a Fuzzy-GA method. International Journal of Electrical Power \& Energy Systems, 30(6-7): pp. 361-367, 2008.

[7]. Sajjadi S.M., Haghifam M.R., and Salehi J., Simultaneous placement of distributed generation and capacitors in distribution networks considering voltage stability index. International Journal of Electrical Power \& Energy Systems, 46: pp. 366-375, 2013.

[8]. Aravindhababu P., and Mohan G., Optimal capacitor placement for voltage stability enhancement in distribution systems. ARPN Journal of Engineering and Applied Sciences, 4(2): pp. 88-92, 2009.

[9]. Devabalaji K., Ravi K., and Kothari D., Optimal location and sizing of capacitor placement in radial distribution system using bacterial foraging optimization algorithm. International Journal of Electrical Power \& Energy Systems, 71: pp. 383-390, 2015.

[10]. Devabalaji K., Yuvaraj T., and Ravi K., An efficient method for solving the optimal sitting and sizing problem of capacitor banks based on cuckoo search algorithm. Ain Shams Engineering Journal, 2016. 
International Journal of Reliability, Risk and Safety:

Theory and Application / Vol. 1, No. 1, 2018

www.IJRRS.com

[11]. Khani H., et al., A new method for online determination of the location of switched capacitor banks in distribution systems. IEEE Transactions on Power Delivery, 26(1): pp. 341-351, 2011.

[12]. Hur K., and Santoso S., On two fundamental signatures for determining the relative location of switched capacitor banks. IEEE Transactions on Power Delivery, 23(2): pp. 1105-1112, 2008.

[13]. Saied M.M., Capacitor switching transients: analysis and proposed technique for identifying capacitor size and location. IEEE Transactions on Power Delivery, 19(2): pp. 759-765, 2004.

[14]. Khodr H., et al., Optimal methodology for distribution systems reconfiguration based on OPF and solved by decomposition technique. European Transactions on Electrical Power, 20(6): pp. 730-746, 2010.

[15]. Khodr H., et al., Maximum savings approach for location and sizing of capacitors in distribution systems. Electric Power Systems Research, 78(7): pp. 1192-1203, 2008.

[16]. Mohan G., and Aravindhababu P., A novel capacitor placement algorithm for voltage stability enhancement in distribution systems. International Journal of Electronics Engineering, 1(1): pp. 83-87, 2009.

[17]. Taher S.A., Hasani M., and Karimian A., A novel method for optimal capacitor placement and sizing in distribution systems with nonlinear loads and DG using $G A$. Communications in Nonlinear Science and Numerical Simulation, 16(2): pp. 851-862, 2011.

[18]. Chis M., Salama M., and Jayaram S., Capacitor placement in distribution systems using heuristic search strategies. IEE Proceedings-Generation, Transmission and Distribution, 144(3): pp. 225-230, 1997.

[19]. Carlisle J., and El-Keib A., A graph search algorithm for optimal placement of fixed and switched capacitors on radial distribution systems. IEEE Transactions on Power Delivery, 15(1): pp. 423-428, 2000.

[20]. De Araujo L.R., et al., Optimal unbalanced capacitor placement in distribution systems for voltage control and energy losses minimization. Electric Power Systems Research, 154: pp. 110-121, 2018.

[21]. Segura S., Romero R., and Rider M.J., Efficient heuristic algorithm used for optimal capacitor placement in distribution systems. International journal of electrical power \& energy systems, 32(1): pp. 71-78, 2010.
[22]. Su C. T., and Tsai C.C.,A new fuzzy-reasoning approach to optimum capacitor allocation for primary distribution systems. in Industrial Technology, 1996.(ICIT'96), Proceedings of The IEEE International Conference on. 1996.

[23]. Jabr R., Optimal placement of capacitors in a radial network using conic and mixed integer linear programming. Electric Power Systems Research, 78(6): pp. 941-948, 2008.

[24]. Short T.A., Electric power distribution handbook. CRC press, 2014.

[25]. BrownR.E., Electric power distribution reliability. CRC press, 2008.

[26]. Pregelj A., Begovic M., and Rohatgi A., Recloser allocation for improved reliability of DG-enhanced distribution networks. IEEE Transactions on Power Systems, 21(3): pp. 1442-1449, 2006.

[27]. Sirjani R., Mohamed A., and Shareef H., Optimal capacitor placement in three-phase distribution systems using improved harmony search algorithm. system, 2(18): pp. 19, 2011.

[28]. Rao R.S., and Narasimham S., Optimal capacitor placement in a radial distribution system using plant growth simulation algorithm. World Academy of Science, Engineering and Technology, 45(120): pp. 715$722,2008$.

[29]. Seifi A., and Hesamzadeh M.R., A hybrid optimization approach for distribution capacitor allocation considering varying load conditions. International Journal of Electrical Power \& Energy Systems, 31(10): pp. 589-595, 2009.

[30].He S., Wu Q.H., and Saunders J., Group search optimizer: an optimization algorithm inspired by animal searching behavior. IEEE transactions on evolutionary computation, 13(5): pp. 973-990, 2009.

[31]. Yan X., Yang W., andShi H., A group search optimization based on improved small world and its application on neural network training in ammonia synthesis. Neurocomputing, 97: pp. 94-107, 2012.

[32]. Mekhamer S.F., Soliman S.A., Moustafa M.A., ElHawar, M. E., "Application of fuzzy logic for reactivepower compensation of radial distribution feeders," IEEE Transactions on Power Systems, Vol. 18, No. 1, pp. 206-213, 2003. 